\title{
Coincidence resolution time of two small scintillators coupled to high quantum-efficiency photomultipliers in a PET-like system
}

\author{
G. Galetta ${ }^{1}$, R. De Leo ${ }^{1,2}$, F. Garibaldi ${ }^{3}$, M.Grodzicka $^{4}$, L. Lagamba ${ }^{1,2}$, F. Loddo $^{2}$, G. Masiello $^{1}$, E. \\ Nappi $^{2}$, R. Perrino ${ }^{5, a}$, A. Ranieri ${ }^{2}$, and T. Szczęśniak ${ }^{4}$ \\ ${ }^{1}$ Dipartimento Interateneo di Fisica "M. Merlin", Bari, Italy \\ ${ }^{2}$ Istituto Nazionale di Fisica Nucleare, Sezione di Bari, Bari, Italy \\ ${ }^{3}$ Istituto Nazionale di Fisica Nucleare, Sezione di Roma, Roma, Italy \\ ${ }^{4}$ National Centre for Nuclear Research (NCBJ) - Otwock-Świerk, Poland \\ ${ }^{5}$ Istituto Nazionale di Fisica Nucleare, Sezione di Lecce, Italy
}

\begin{abstract}
The lower limit of the time resolution for a positron emission tomography (PET) system has been measured for two scintillator types, LYSO:Ce and LuAG:Pr. Small dimension crystals and ultra bi-alkali phototubes have been used in order to increase the detected scintillation photons. Good timing resolutions of 118 ps and 223 ps FWHM have been obtained for two LYSO and two LuAG, respectively, exposed to a ${ }^{22} \mathrm{Na}$ source.
\end{abstract}

\section{Introduction}

Lowering of the coincidence resolution time (CRT) in the detection of the two $511 \mathrm{keV} \gamma$-rays from $e^{+} e^{-}$annihilation events considerably improves the signal-to-noise ratio of PET devices [1]. The timing performance of a scintillator-detector system is caused by many parameters, some related to the scintillator (rise time, decay time, optical photon yield, geometry, and surface finish), some to the photodetector (quantum efficiency QE, transit time spread TTS, single electron response SER). A further contribution derives from time pick-off methods, e.g. leading edge (LED) or constant fraction discrimination (CFD) and full digitization techniques.

In the present work we have investigated the lower CRT value that can be obtained by using recent photomultipliers (PMTs) from Hamamatsu with higher QE values, and the most used scintillator in standard PET, Proteus LYSO crystals. For comparison the CRT has also been measured for Furukawa LuAG:Pr scintillators. Contributions from optical photons propagation [2] have been minimized by using small size crystals, at the cost of a low detection efficiency. The LED method has been adopted because it performs [3] the best CRT value at lower thresholds with usual laboratory electronics.

\section{Materials, methods and measurements}

Two metal package ultra bi-alkali (UBA) R7600U-200 PMTs with the E5996 resistor chain from Hamamatsu Photonics have been used $(\mathrm{QE}=43 \%$ @ $350 \mathrm{~nm}$, rise time $1.4 \mathrm{~ns})$. The PMTs were

a e-mail: roberto.perrino@le.infn.it

This is an Open Access article distributed under the terms of the Creative Commons Attribution License 2.0, which permits unrestricted use, distribution, and reproduction in any medium, provided the original work is properly cited. 
operated at the maximum allowed voltage $(900 \mathrm{~V})$ in order to minimize the TTS (TTS $=350 \mathrm{ps})$. The PMTs have been characterized with a $409 \pm 1 \mathrm{~nm}$ pico-laser (PIL040SM from A.L.S. GmbH with $\sigma_{t}=$ 20 ps pulse duration. The PMTs signals in the single photoelectron regime $\left(Q_{1 p e}\right)$ (attenuated laser), pre-amplified by a CAEN V978, were integrated in a charge ADC gated by the laser trigger. The TTS was measured in a TDC started by the same laser trigger and stopped by the LED set at a very low threshold $(2 \mathrm{mV})$. The associated noise was $\sigma_{r m s}=1.5 \mathrm{mV}$. At half pulse height, an average SER slope $d V_{1} / d t=17 \mathrm{mV} / \mathrm{ns}$ was measured. Single photon charge $Q_{1 p e}=1.25 \mathrm{pC}$ with $\sigma_{Q 1 p e}=0.64$ $\mathrm{pC}$ was measured, yielding an excess noise factor $\mathrm{ENF}=1.26$. Time-walk corrected (owing to the charge-time correlated measurements) TTS values were 265 ps and 390 ps for the two PMTs, in good agreement with Hamamatsu specifications (350 ps).

CRTs were measured for two $3 \times 3 \times 5 \mathrm{~mm}^{3}$ LYSO and two $3 \times 3 \times 3 \mathrm{~mm}^{3}$ LuAG:Pr crystals, respectively. All crystal surfaces were optically polished, with five surfaces wrapped in many layers of Teflon. The experimental set up includes two couples of back-to-back scintillator+PMT pointing towards a ${ }^{22} \mathrm{Na}$ source, plus NIM/CAMAC signal processing electronics. Each PMT signal is resistively split to get charge and time information. The time is picked up at a given threshold Q in a CAEN N841 LED. The event trigger is defined by the coincidence of signals over threshold in both detector arms. This also provides the start for a CAEN C414 TDC with 25 ps resolution and the integration gate for a LeCroy $2249 \mathrm{~W}$ ADC with $0.25 \mathrm{pC} /$ count. TDC time stops were taken from the two individual discriminated signals, suitably delayed to enter the TDC full scale.

Signals for both scintillators (Fig. 1a) at the $511 \mathrm{keV}$ photopeak from ${ }^{22} \mathrm{Na}$ show different average heights $\left(V_{511 \mathrm{keV}}=5 \mathrm{~V}\right.$ for LYSO, $2.6 \mathrm{~V}$ for LuAG) and decay times [4] $\left(T_{d}^{511 \mathrm{keV}}=40 \mathrm{~ns}\right.$ for LYSO [5], $20 \mathrm{~ns}$ for LuAG [6]), but display comparable rise times $\left(T_{r}^{511 \mathrm{keV}}=4.4 \mathrm{~ns}\right.$ for LYSO, 4.2 for LuAG), mostly dominated by PMT and electronics shaping.

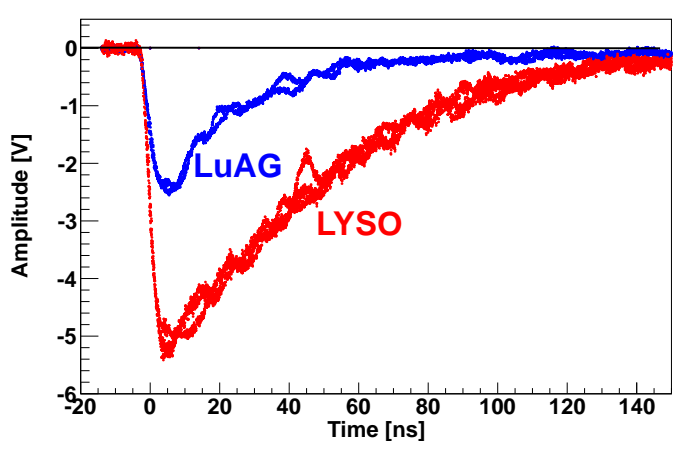

(a)
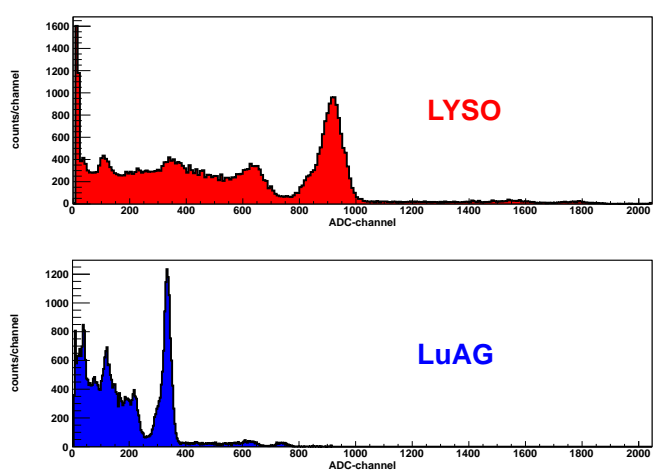

(b)

Figure 1: (a) R7600U-200 PMT waveforms for $511 \mathrm{keV}$ photopeak from ${ }^{22} \mathrm{Na}$ source exciting the two studied crystals. (b) Corresponding whole charge spectra of $\gamma$-rays measured from ${ }^{22} \mathrm{Na}$.

The ratio of the charge for the $511 \mathrm{keV}$ signals to the single photoelectron charge $Q_{1 p e}$, measured from SER, provides the photoelectron ( $p e$ ) yield $N_{p e}=4240 \pm 150$ for LYSO [7] and $N_{p e}=1727 \pm 182$ for LuAG [8] crystals, respectively. The different $N_{p e}$ values are due to the different scintillator light yields and peak emission ( $310 \mathrm{~nm}$ for LuAG, and $420 \mathrm{~nm}$ for LYSO). Spectra from these two crystals exposed to $\gamma$-rays from a ${ }^{22} \mathrm{Na}$ source are displayed in Fig. 1b. The energy resolutions for the $511 \mathrm{keV}$ photopeaks are: $\sigma / \mathrm{E}=3.16 \%$ for LYSO, and $\sigma / \mathrm{E}=3.38 \%$ for LuAG. After the quadratic subtraction of the statistical contribution $\left.(\sigma / E)_{\text {stat }}=\sqrt{E N F / N_{p e}}\right)$, the intrinsic resolution $(\sigma / E)_{\text {intr }}^{511 \mathrm{keV}}$ quotes $2.65 \%$ for LYSO, and $2.03 \%$ for LuAG. 
INPC 2013

\section{Coincidence time resolution results}

The charge correlation plot (Fig. 2a) provides the accurate selection of coincident photoelectric events of $511 \mathrm{keV} \gamma$-rays from ${ }^{22} \mathrm{Na}$ in both crystals against Compton, intrinsic radioactivity and higher energy radiation from source.

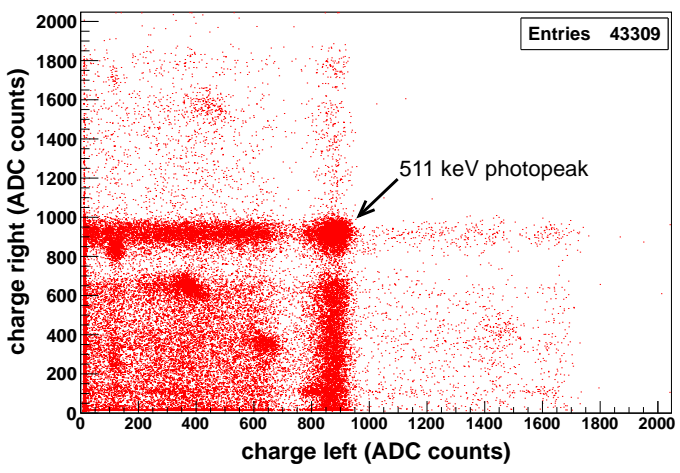

(a)

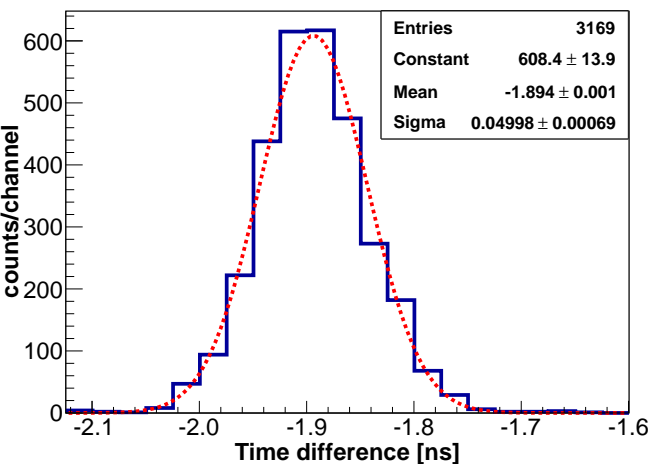

(b)

Figure 2: (a) Correlation of signal charges for the two PMT+LYSO detectors with ${ }^{22} \mathrm{Na}$ source in between. (b) Gaussian fitted time difference spectrum of correlated $511 \mathrm{KeV} \gamma$-rays detected in LYSO crystals, at Q-threshold $=5 \mathrm{mV}$, yielding $\sigma_{C R T}=50 \mathrm{ps}(\mathrm{FWHM}=118 \mathrm{ps})$ time resolution.

The spectrum (Fig. 2b) of time differences between the two detector arms, gated on photoelectric effect (arrow in Fig. 2a), measures the CRT timing. Their standard deviations $\sigma_{C R T}$ were measured at several discrimination thresholds (Q) for both LYSO and LuAG crystals. The $\sigma_{C R T}$ as a function of $\mathrm{Q}$ (Fig. 3) is generally explained by the quadratic sum of independent contributions from scintillators, photodetectors, signal transport, and handling electronics. The electronics contribution $\sigma_{0}$, measured by the time spread (16 ps) of the TDC start signal, weakly affects the overall measured resolutions. The scintillation contribution $\sigma_{p h o t}$ due to the underlying photon production statistics, depends on the scintillators. If written as $\sigma_{p h o t}=c_{1} \sqrt{\left(Q T_{r} T_{d} / N_{p e}\right)}$, it accounts for the high-Q values of $\sigma_{C R T}$. Q is expressed in pe, $1 p e \approx 11 \mathrm{mV}$. The constant $c_{1}$ should be the same for both scintillators and depends on the PMT. The contribution of photodetector noise is $\sigma_{\text {noise }}=\sigma_{r m s} /(d V / d t)_{Q}$, where $(d V / d t)_{Q}$ is the signal slope at threshold Q, and the $\sigma_{T T S}=(T T S / 2.35) / \sqrt{N_{p e Q}}$, being $N_{p e Q}$ the number of detected $p e$ at threshold Q. Both these strongly influence data at low-Q values with a $1 / \sqrt{Q}$ dependence, and a constant value at high Q-thresholds. The data in Fig. 3 have therefore been fitted by the combination $\sigma_{C R T}(Q)=c_{1} \sqrt{\left(Q T_{r} T_{d} / N_{p e}\right)} \oplus c_{2} / \sqrt{N_{p e}} \oplus c_{3} / \sqrt{N_{p e} Q}$, with the same value of the three constants for both scintillators: $c_{1}=(0.82 \pm 0.02), c_{2}=(2.2 \pm 0.3) n s \cdot \sqrt{p e}, c_{3}=(1.8 \pm 0.5) n s \cdot p e$.

\section{Summary}

The CRT values of two LYSO and two LuAG:Pr scintillators in a PET-like geometry have been measured. Small size crystals have been employed in order to neglect the contribution from light transport. Two R7600U-200 PMTs with UBA photocathode have been used. The PMTs have preliminarily been characterized with a short-pulse pico-laser. Their performances agree with Hamamatsu specifications. Owing to UBA high QE PMTs and to the small crystal sizes, a high number of pe is measured for 511 


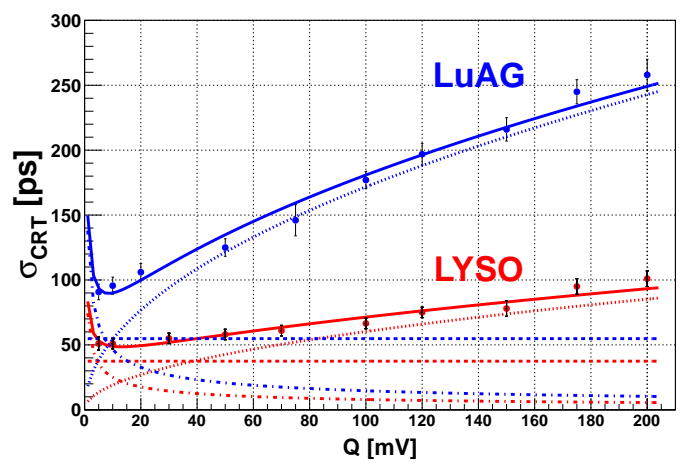

Figure 3: The measured CRT standard deviation timing resolutions, $\sigma[p s]$, at different LED discriminator thresholds $Q[m V]$ the for LYSO (blue) and LuAG (red) scintillators. Full curves are the complete fit. Dotted (statistics) and dash-dotted curves are the individual contributions as in the text.

$\mathrm{keV} \gamma$-rays $\left(N_{p e}=4240\right.$ in LYSO, 1727 in LuAG). The time information from the two PMTs has been derived by using the LED technique. With PMTs at the maximum allowed voltage, we have measured CRT at LED thresholds as low as $Q \approx 0.5$ pe. Our CRT data confirm a well-known conclusion: a high pe detection is the easiest way to reach good CRT values. CRTs increase with Q. At low Q, CRT is mostly influenced by the photodetector (TTS, noise) performance, while, at higher Q CRTs strongly depend on the scintillation statistics. In this last case both rise and decay times of the scintillator contribute. The best FWHM CRT values, here obtained at $Q \approx 0.5$ pe, are 118 ps for LYSO and 223 ps for LuAG.

These results, performed with conventional electronics, are promising and comparable with current literature for these scintillators. In similar conditions, some authors [9] obtain CRT values of about 200 ps with photomultipliers. With different photodetectors, i.e. Silicon photomultipliers, some authors [5] report 137 ps FWHM, others [10] 108 ps FWHM for slightly smaller $\left(2 \times 2 \times 3 \mathrm{~mm}^{3}\right)$ crystals. In both cases they have used also fast waveform sampling techniques.

UBA PMTs and small crystal size are the main ingredients for the results here reported, even if small crystals necessarily deal with low detection efficiencies. Real PET devices necessarily have to face with the well-known timing-efficiency tradeoff.

\section{References}

[1] S. Ziegler et al., IEEE Trans. on Nucl. Sci., 37, 574-579 (1990)

[2] W.S. Choong, Phys. Med. Biol., 54, 6495-6513 (2009)

[3] T. Szczęśniak et al., IEEE Trans. on Nucl. Sci., 54, 1464-1473 (2007)

[4] M. Conti et al., IEEE Trans. on Nucl. Sci., 56, 926-933 (2009)

[5] S. Seifert et al., JINST, 7, P09004 (2012)

[6] K. Kamada et al., IEEE Trans. on Nucl. Sci., 58, 570-573 (2009)

[7] J.T.M. de Haas et al., IEEE Trans. on Nucl. Sci., 55, 1086-1092 (2008)

[8] W. Drozdowski et al., IEEE Trans. on Nucl. Sci., 55, 2420-2424 (2008)

[9] M. Moszynski et al., Nucl. Instr. Meth., A567, 31-35 (2006)

[10] S. Gundacker et al., Nucl. Instr. Meth., A, in press, available on line 